\title{
NUMERO DI CASI CONFERMATI DI SIFILIDE NELLE DONNE INCINTE IN BRASILE TRA IL 2009 E IL 2013
}

\section{ARTICOLO ORIGINALE}

BARROS, Yara Lorrane Souza de ${ }^{1}$, FECURY, Amanda Alves², OLIVEIRA, Euzébio de $^{3}$, DENDASCK, Carla Viana ${ }^{4}$, ARAÚJO, Maria Helena Mendonça de ${ }^{5}$, SOUZA, Keulle Oliveira da ${ }^{6}$, DIAS, Claudio Alberto Gellis de Mattos ${ }^{7}$

BARROS, Yara Lorrane Souza de. Et. Numero di casi confermati di sifilide nelle donne incinte in Brasile tra il 2009 e il 2013. Revista Científica Multidisciplinar Núcleo do Conhecimento. Anno 05, Ed. 11, Vol. 25, pp. 53-61. novembre 2020. ISSN: 2448-0959, Link di accesso: https://www.nucleodoconhecimento.com.br/salute/donne-incinte, DOI: 10.32749/nucleodoconhecimento.com.br/salute/donne-incinte

\section{RIEPILOGO}

La sifilide è una malattia batterica trasmessa sessualmente dalle donne incinte con sifilide al feto. La diagnosi di sifilide nelle donne in gravidanza è confermata dall'esame VDRL. L'obiettivo di questo lavoro è mostrare il numero di casi confermati di sifilide nelle donne incinte in Brasile, come anno di diagnosi, fascia d'età, regioni del Brasile, zona residenziale, tipo di test, classificazione ed evoluzione tra il $2009 \mathrm{e}$ il 2013. I dati secondari sono stati utilizzati dal reparto computer di SUS - DATASUS (http://datasus.saude.gov.br). II maggior numero di test consente un trattamento

\footnotetext{
${ }^{1}$ Tecnica mineraria, come risultato dell'Istituto Federale di Amapá (IFAP).

2 Biomedicale, Dottorato di Ricerca in Malattie Tropicali, Professore e ricercatore del Corso di Medicina dell'Università Federale di Amapá (UNIFAP)

${ }^{3}$ Biologo, Dottore di Ricerca in Malattie Topiche, Professore e ricercatore del Corso di Educazione Fisica dell'Università Federale di Pará (UFPA).

${ }^{4}$ Teologo, Dottore di Ricerca in Psicoanalisi, ricercatore presso il Centro di Ricerca e Studi Avanzati - CEPA.

${ }^{5}$ Medico, professore e ricercatore del corso di medicina dell'Università federale di Amapá (UNIFAP).

${ }^{6}$ Sociologo, studente magistrale in Studi Antropici in Amazzonia, Membro del Gruppo di Ricerca "Laboratorio di Educazione, Ambiente e Salute" (LEMAS/UFPA).

${ }^{7}$ Biologo, Dottore di Ricerca in Teoria e Comportamento, Professore e ricercatore del Graduate Program in Professional and Technological Education (PROFEPT), Istituto Federale di Amapá (IFAP).
}

RC: 68388

Disponibile in: https://www.nucleodoconhecimento.com.br/salute/donne-incinte 
migliore e una riduzione del numero di casi. Tuttavia, l'assenza di cure preventive durante le relazioni sessuali induce un aumento dei casi, specialmente tra le donne in età riproduttiva. Le aree con una popolazione più numerosa hanno un numero maggiore di casi. L'elevata variazione di partner in queste aree determina una maggiore contaminazione. II tipo di test influenza i numeri. Test meno specifici e più generali trovano anticorpi specifici e non specifici per il batterio. Questo può cambiare i numeri effettivi. L'assistenza sanitaria femminile rende precoce la diagnosi, facilitando il trattamento. La sifilide non è direttamente responsabile del maggior numero di mortalità nelle donne in gravidanza.

Parole chiave: Epidemiologia, sifilide, donne incinte.

\section{INTRODUZIONE}

La sifilide è una malattia batterica che colpisce tutto il corpo, trasmessa sessualmente; mediante donazione di sangue; contatto diretto con sangue contaminato; e la donna incinta con sifilide per il feto. II batterio che causa la malattia è Treponema Pallidum (CARVALHO et al., 2014, JUNIOR et al., 2009; ROCHA et al., 2020).

I sintomi della sifilide si manifestano in tre fasi. Nella prima fase possono apparire macchie rosse sul corpo, ferite di solito sui genitali. Nella seconda fase è possibile che la malattia sia dormiente per un periodo massimo di otto mesi, quando il batterio può essere distribuito interessando la pelle e gli organi. Nella terza fase è comune apparire infiammazioni nei tessuti del corpo, come ossa, muscoli e fegato (AVELLEIRA et al., 2006; BRETAS et al., 2008).

La malattia deve essere diagnosticata in modo che il paziente possa fare il trattamento appropriato, prevenendo la trasmissione della malattia attraverso l'uso di preservativi maschi / femmine. II trattamento più efficace è il farmaco benzaycin penicillina. Fino al 2015 non c'era traccia di casi di resistenza ad esso. Altri farmaci

$\mathrm{RC}: 68388$

Disponibile in: https://www.nucleodoconhecimento.com.br/salute/donne-incinte 
sono usati nel trattamento della sifilide, ma quello che ha la massima efficienza nel trattamento / cura è questo tipo di penicillina (KALININ et al., 2015; BRASIL, 2015).

La diagnosi di sifilide nelle donne in gravidanza è confermata dall'esame VDRL (Venereal Disease Research Laboratory) durante le cure prenatali. La scoperta della malattia e il trattamento delle donne in gravidanza durante le cure prenatali con penicillina prevengono la sifilide congenita (SUTO et al., 2016; MESQUITA et al., 2012).

II numero di casi di sifilide nelle donne incinte in Brasile è stato di 21382 nel 2012 e nel 2013 (BRASIL 2015).

II numero di casi di sifilide nelle donne incinte nella regione nord-orientale è stato di 4.433 nel 2012 e nel 2013. Nello stesso periodo, il numero di casi di sifilide nelle donne incinte nella regione sud-orientale era di 10.052; nella regione meridionale era il 2795; nel Midwest era il 1961; e nella regione del Nord era 214 (BRASIL, 2015).

II numero di casi di sifilide nelle donne incinte ad Amapá è stato di 176 nel 2012 e nel 2013 (BRASIL 2015).

\section{GOL}

Lo scopo di questo articolo è quello di mostrare il numero di casi confermati di sifilide nelle donne incinte in Brasile, come l'anno di diagnosi, fascia d'età, regioni del Brasile, zona residenziale, tipo di test, classificazione ed evoluzione tra il 2009 e il 2013.

\section{METODO}

Dati presi dal reparto computer di SUS - DATASUS (http://datasus.saude.gov.br) seguendo i seguenti passaggi: in primo luogo, è stata selezionata la scheda "accesso alle informazioni", seguita dall'opzione "informazioni sanitarie (TABNET)" subito dopo l'opzione "epidemiologica e morbilità". Ha cliccato sull'opzione "Malattie

RC: 68388

Disponibile in: https://www.nucleodoconhecimento.com.br/salute/donne-incinte 
e malattie notificabili dal 2007 in poi (SINAN)" e poi "Sifilide in gravidanza", e nella scheda "copertura geografica" selezionata "Brasile per regione, UF e comune". I passaggi successivi sono stati: A) nella riga selezionata "Casi confermati in base all'anno di diagnosi", nella colonna "non attivo" e nel contenuto "casi confermati". Per questa opzione e per i seguenti dati sono stati raccolti dal 2009 al 2013. B) nella riga, sono stati selezionati "Casi confermati in base all'area di notifica", nella colonna "non attivi" e nel contenuto "casi confermati". C) nella riga, sono stati selezionati "Casi confermati in base alla fascia d'età", nella colonna "non attivi" e nel contenuto "casi confermati". D) nella riga "Casi confermati secondo Evolution", nella colonna "non attivo" e nel contenuto sono stati selezionati "casi confermati". E) nella riga, sono stati selezionati "Casi confermati secondo la classificazione clinica", nella colonna "non attivi" e nel contenuto "casi confermati". F) nella riga "Casi confermati secondo test non Trep", nella colonna "non attivo" e nel contenuto sono stati selezionati "casi confermati". G) nella riga "Casi confermati secondo Trep Test" sono stati selezionati nella colonna "non attivi", in contenuti "casi confermati". H) nella riga selezionare "Casi confermati per residenza di zona secondo anno di diagnosi", nella colonna selezionare "Zona residenziale" e nel contenuto "casi confermati". I dati sono stati compilati all'interno dell'applicazione Excel, un componente della suite Microsoft Corporation Office. La ricerca bibliografica è stata condotta in articoli scientifici, utilizzando computer del laboratorio informatico dell'Istituto Federale di Educazione, Scienza e Tecnologia di Amapá, Macapá Campus, situato a: Rodovia BR 210 KM 3, s/n - Bairro Brasil Novo. CEP: 68.909-398, Macapá, Amapá, Brasil.

\section{RISULTATI}

La figura 1 mostra il numero di casi confermati di sifilide nelle donne incinte all'anno in Brasile tra il 2009 e il 2013. I dati mostrano un aumento del numero di casi confermati dal 2009 al 2012. Nel 2013 tale numero è diminuito della metà rispetto all'anno precedente.

RC: 68388

Disponibile in: https://www.nucleodoconhecimento.com.br/salute/donne-incinte 
Grafico 1 Numero di casi confermati di sifilide nelle donne incinte all'anno in Brasile tra il 2009 e il 2013.

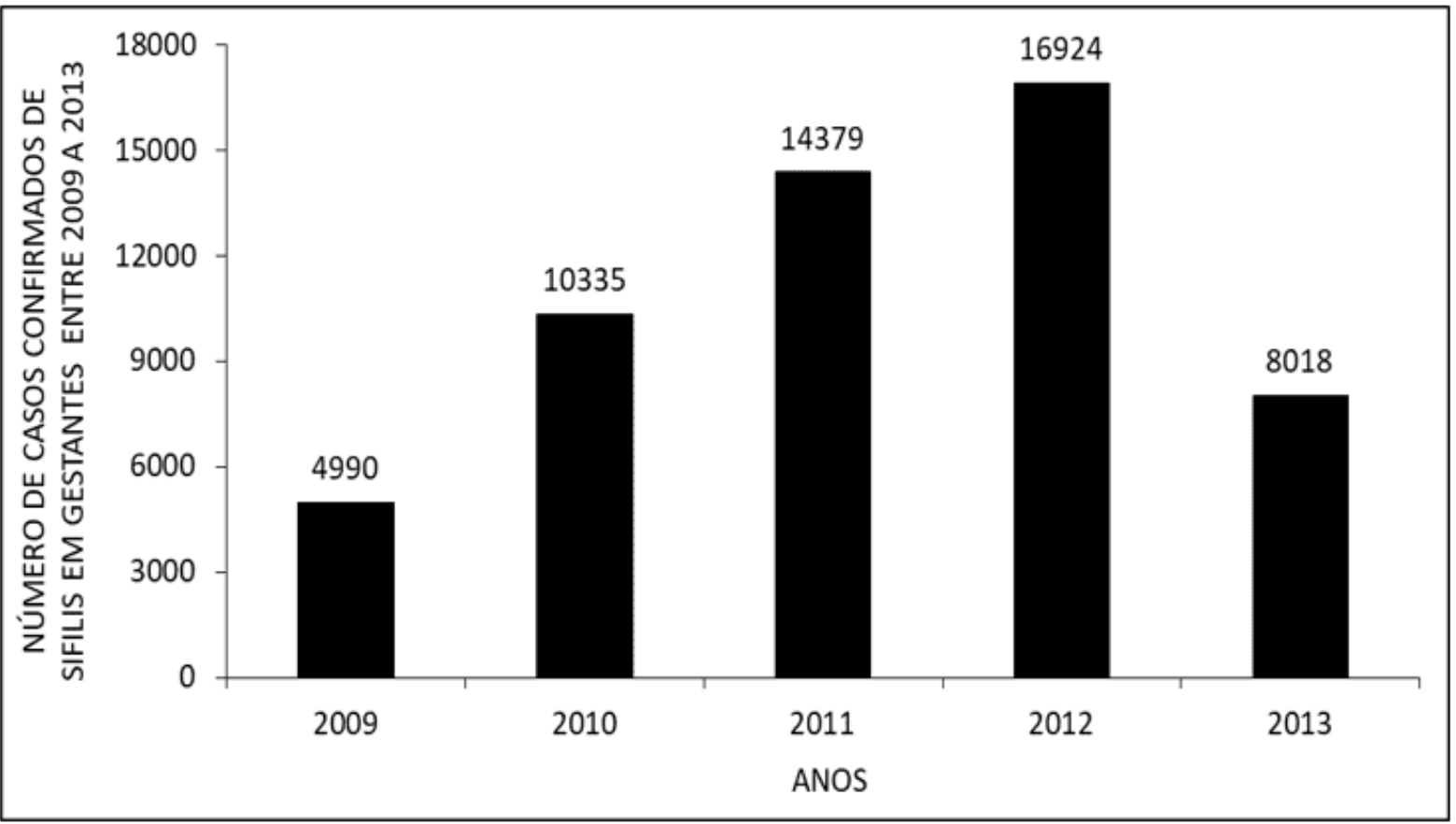

La figura 2 mostra il numero di casi confermati di sifilide nelle donne incinte per fascia d'età in Brasile tra il 2009 e il 2013. Tra i 10 e i 39 anni c'è stata una crescita. Questo numero diminuisce tra i 40 e i 64 anni. 
Grafico 2 Numero di casi confermati di sifilide nelle donne incinte per fascia d'età in Brasile tra il 2009 e il 2013.

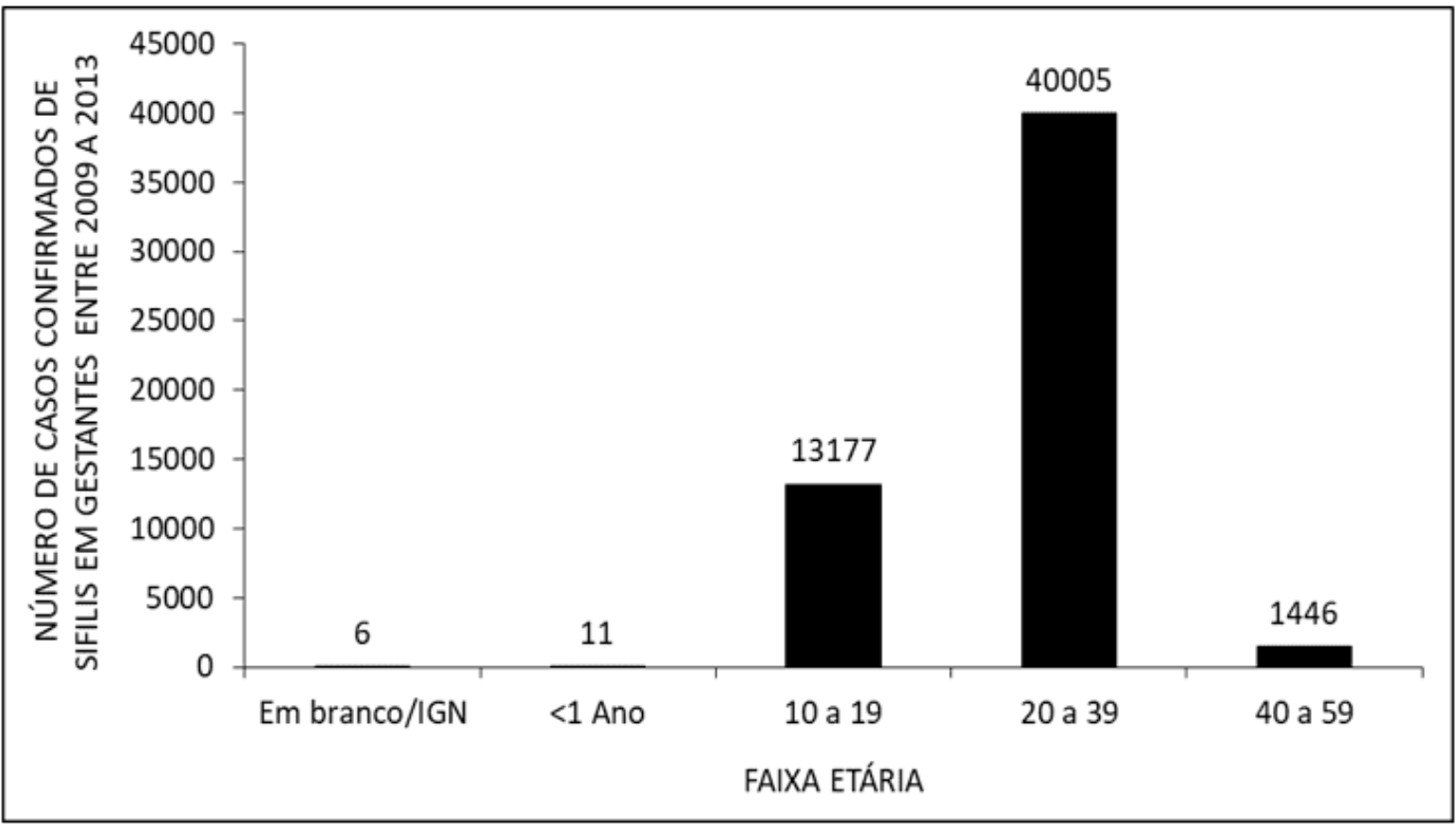

La figura 3 mostra il numero di casi confermati di sifilide nelle donne incinte per regione in Brasile tra il 2009 e il 2013. La regione sud-orientale ha il maggior numero di casi confermati, seguita rispettivamente dalle regioni nord-est, nord, sud e midwest.

RC: 68388

Disponibile in: https://www.nucleodoconhecimento.com.br/salute/donne-incinte 
Grafico 3 Numero di casi confermati di sifilide nelle donne incinte per regione del paese in Brasile tra il 2009 e il 2013.

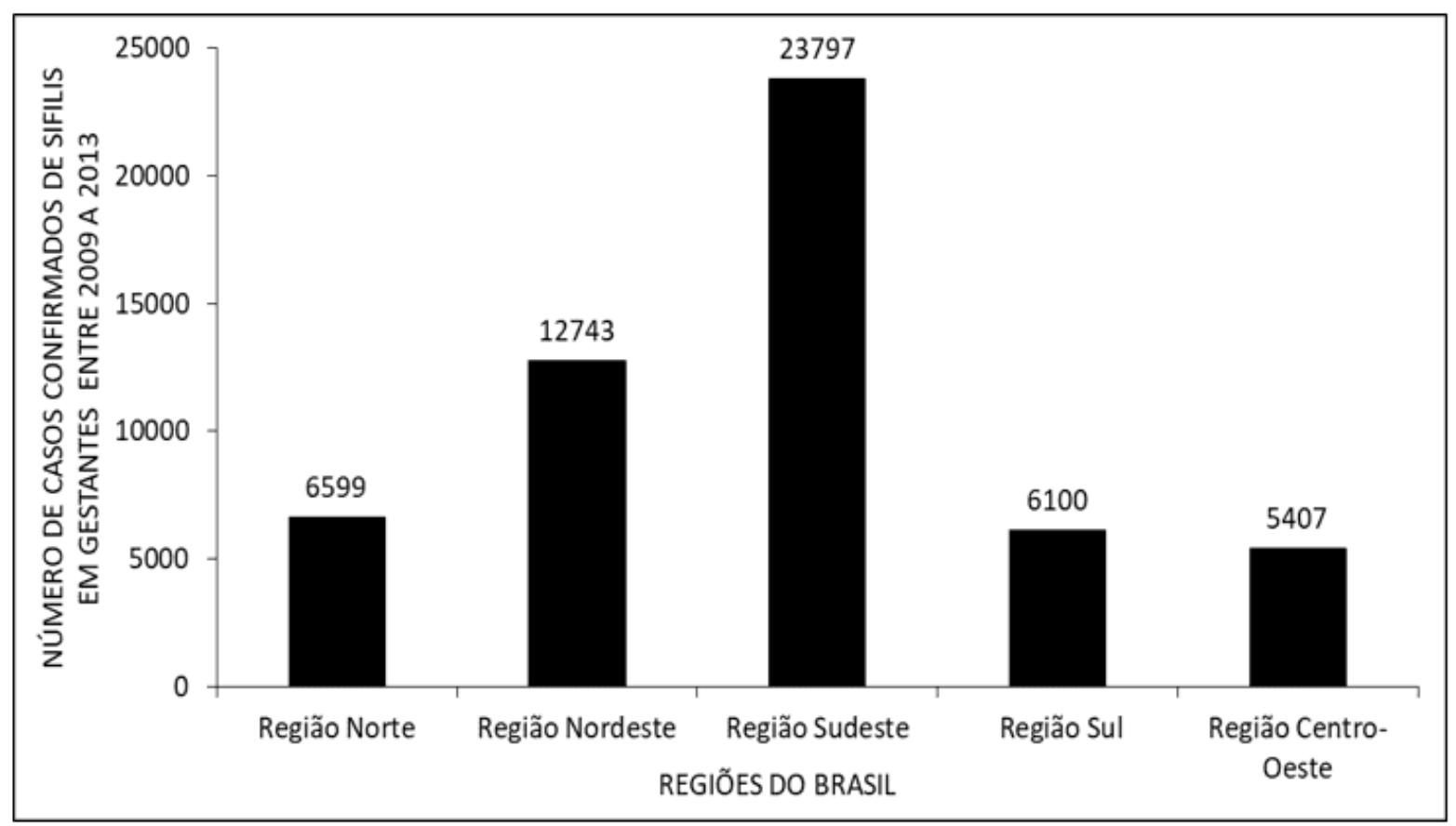

La figura 4 mostra il numero di casi confermati di sifilide nelle donne incinte per zona di residenza in Brasile tra il 2009 e il 2013. II numero nell'area urbana è circa 8 volte superiore a quello dell'area rurale. II numero più basso di casi per area di residenza è rispettivamente periurbano e ign/branco.

RC: 68388

Disponibile in: https://www.nucleodoconhecimento.com.br/salute/donne-incinte 
Grafico 4 Numero di casi confermati di sifilide nelle donne incinte in base al tipo di area di residenza in Brasile tra il 2009 e il 2013.

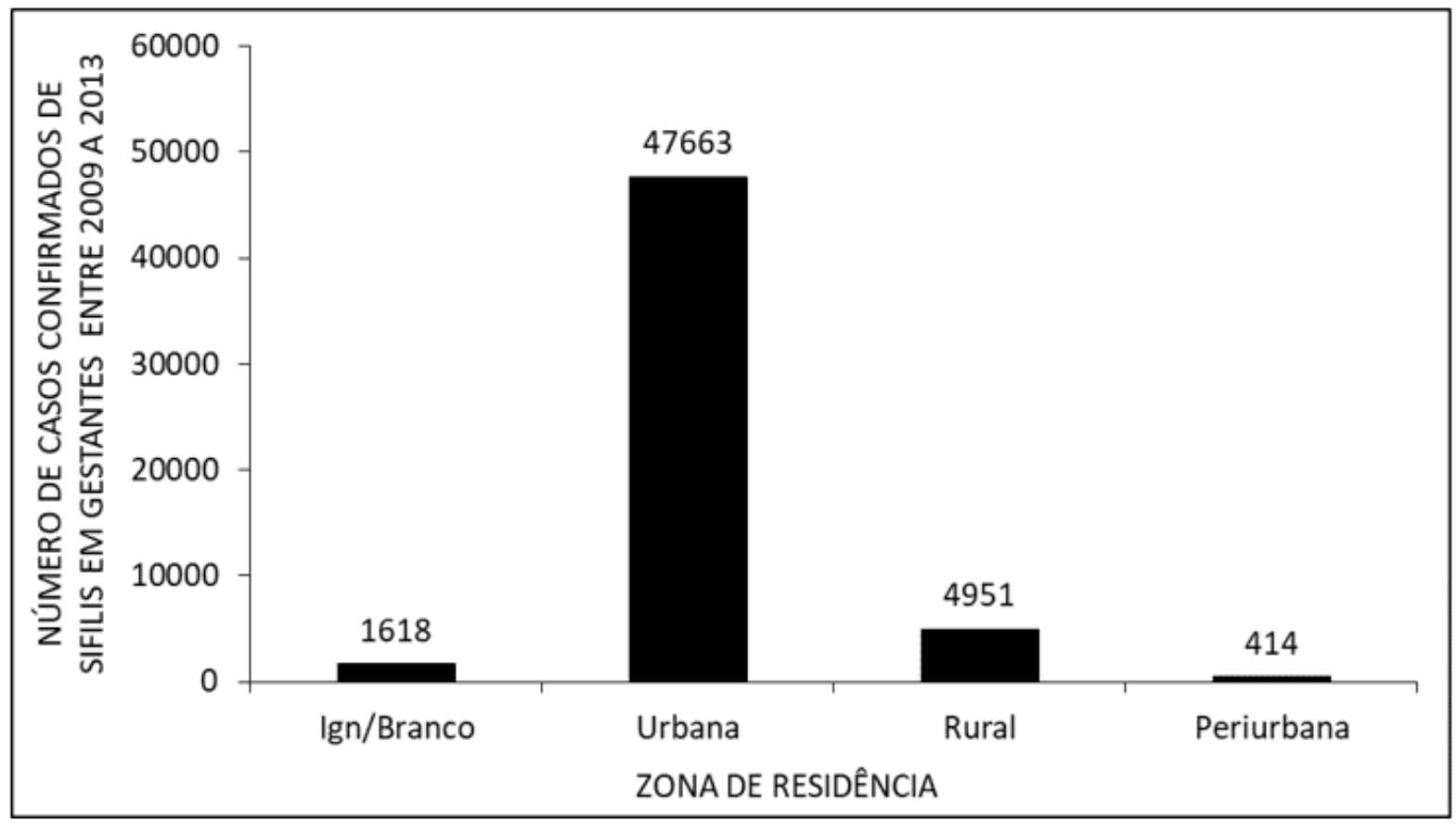

La figura 5 mostra il numero di casi confermati di sifilide nelle donne incinte per tipo di test eseguito in Brasile tra il 2009 e il 2013. II numero di casi di test ign/white eseguiti per test TREP è un frame times superiore ai test NON-TREP. II numero di casi reattivi da parte dei test NON-TREP è quasi il doppio dei casi reattivi dai test TREP. II numero di casi non reattivi tra test NON-TREP e TREP è approssimativo. II numero di casi non eseguiti di test TREP è nove volte superiore al numero di casi non eseguiti da test NON TREP.

RC: 68388

Disponibile in: https://www.nucleodoconhecimento.com.br/salute/donne-incinte 
Figura 5 Numero di casi confermati di sifilide nelle donne incinte in base al tipo di test eseguito in Brasile tra il 2009 e il 2013.

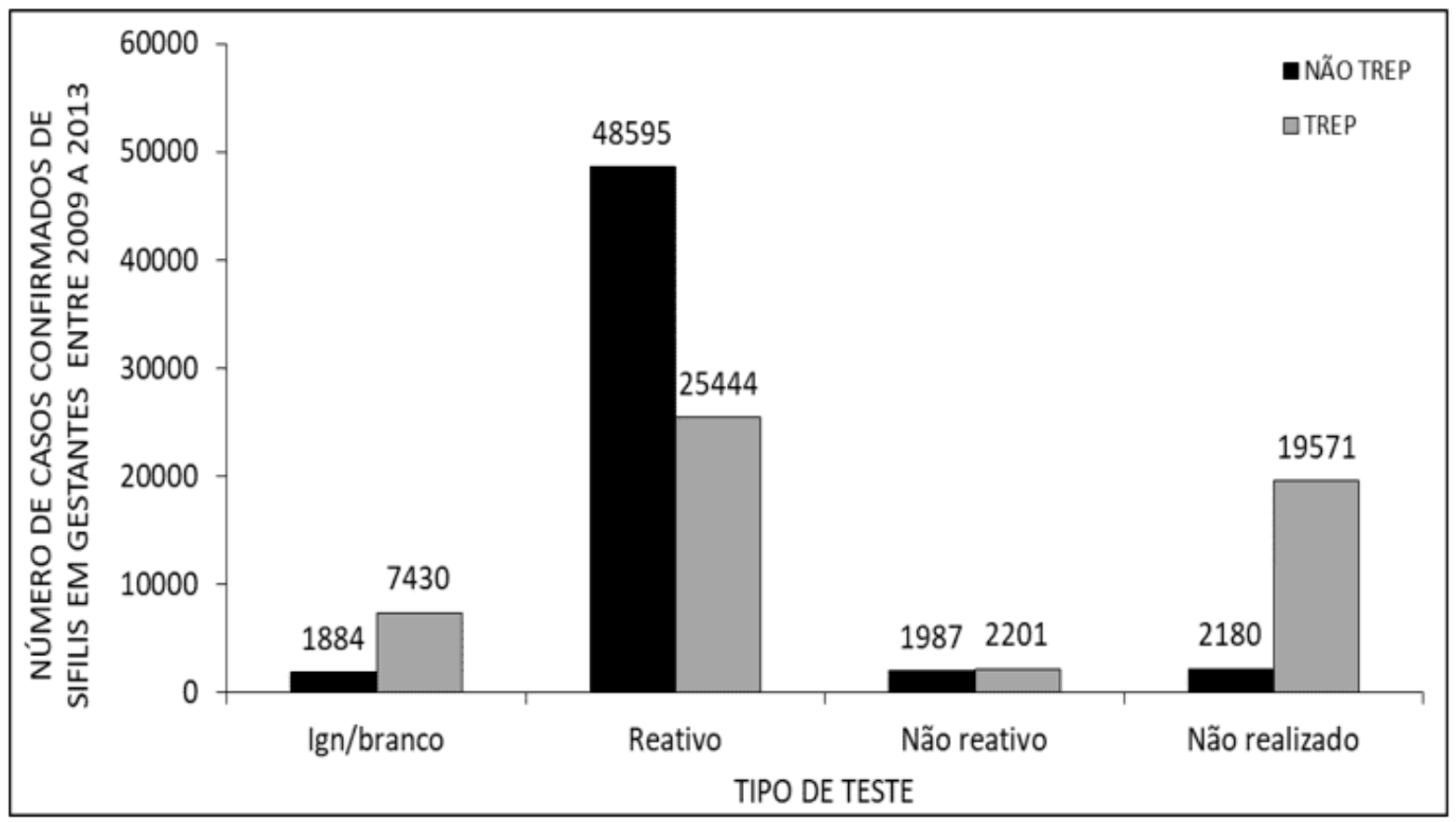

La figura 6 mostra il numero di casi confermati di sifilide nelle donne incinte secondo la classificazione in Brasile tra il 2009 e il 2013. La classificazione primaria ha il maggior numero di casi, seguiti rispettivamente da Ign/White e latente. Le classificazioni, secondaria e terziaria, hanno il minor numero di casi confermati.

RC: 68388

Disponibile in: https://www.nucleodoconhecimento.com.br/salute/donne-incinte 
Figura 6 Numero di casi confermati di sifilide nelle donne incinte secondo la classificazione in Brasile tra il 2009 e il 2013.

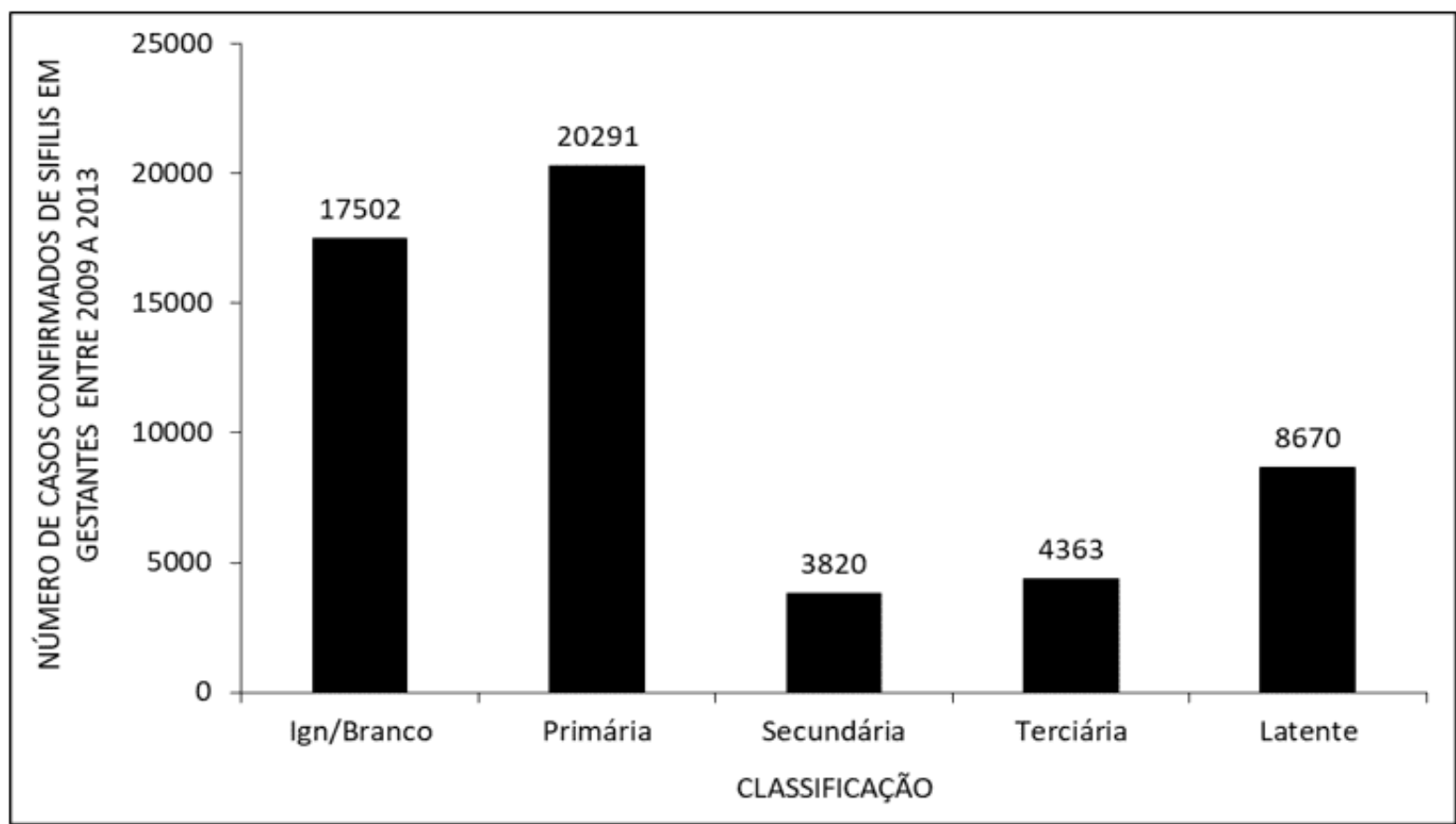

La figura 7 mostra il numero di casi confermati di sifilide nelle donne incinte secondo l'evoluzione in Brasile tra il 2009 e il 2013. I dati mostrano che il numero di decessi di donne incinte che hanno acquisito sifilide in questo periodo è stato il doppio del numero di cure. II maggior numero di morti si è verificato a causa di altre cause. II numero più basso di decessi nel periodo è apparso come indagato.

RC: 68388

Disponibile in: https://www.nucleodoconhecimento.com.br/salute/donne-incinte 
Grafico 7 Numero di casi confermati di sifilide nelle donne incinte secondo l'evoluzione in Brasile tra il 2009 e il 2013.

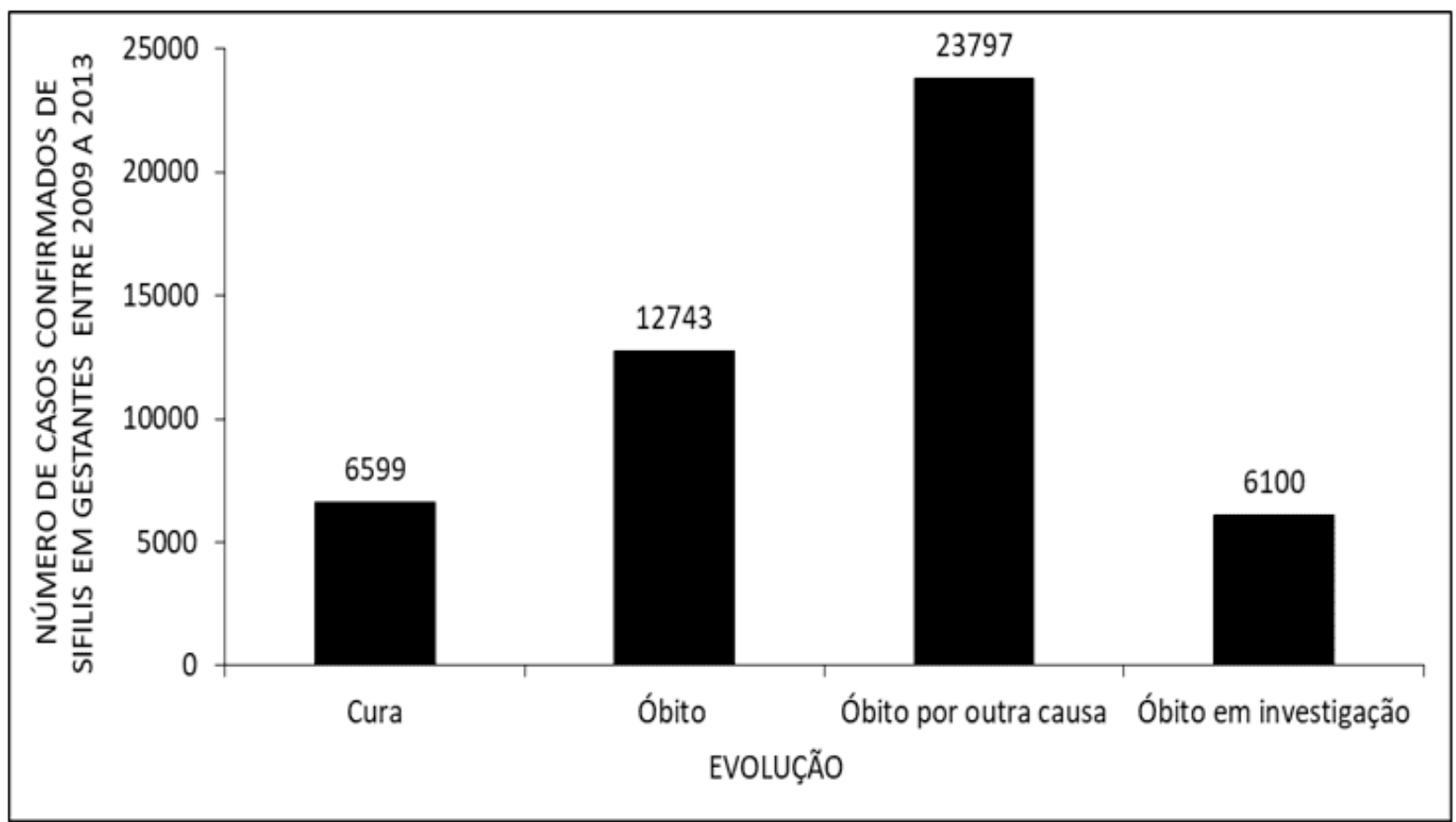

\section{DISCUSSIONE}

Tra il 2009 e il 2012 si è registrato un aumento del numero di casi confermati di sifilide nelle donne incinte. Tra il 2011 e il 2013 si è registrato un aumento del numero di test, da 31,5 mila a 1,7 milioni, che sembra aver permesso di curare il paziente con sifilide. Questo scenario potrebbe aver influenzato il calo dei casi confermati nelle donne incinte nel 2013 (BRASIL, 2013; 2017).

I dati mostrano che i casi di sifilide nelle donne incinte sono aumentati tra i 10 e i 39 anni e la sua diminuzione tra i 40 e i 64 anni (figura 2), corroborata dalla letteratura. Nella fascia di età compresa tra i 20 e i 34 anni, le donne sono al culmine della loro fase riproduttiva. Sembra esserci un tasso più elevato di donne incinte con sifilide in questa fascia di età, probabilmente spiegato dalla pratica del sesso non protetto (COSTA et al, 2012).

RC: 68388

Disponibile in: https://www.nucleodoconhecimento.com.br/salute/donne-incinte 
Il sud-est è la regione brasiliana con il maggior numero di casi di sifilide nelle donne incinte nel periodo esaminato. Questi dati possono essere spiegati dal fatto che il sud-est concentra la maggior parte della popolazione brasiliana e circa la metà della popolazione ammette di non usare preservativi nelle relazioni sessuali (BRASIL, 2011; IBGE, 2010).

Nel periodo studiato, l'area urbana del Brasile ha presentato un numero più elevato di casi di donne incinte con sifilide rispetto all'area rurale (figura 4). Secondo la letteratura, le persone della popolazione urbana sono sessualmente imparentate con un maggior numero di partner. Ciò può portare a un aumento della probabilità che un individuo senza un partner sessuale stabile o fisso acquisisca infezioni sessualmente trasmissibili come la sifilide (BRASIL, 2011).

II maggior numero di casi confermati è stato nei test NON-TREP che nei test TREP. Ciò è probabilmente dovuto al fatto che i test TREP sono più specifici individuando solo anticorpi contro il batterio, mentre $i$ test NON-TREP individuano anticorpi specifici e non specifici del batterio Treponema pallidum (BRASIL, 2015).

C'è stato un alto numero di casi confermati di sifilide nelle donne incinte per classificazione primaria. Si presume che dopo il primo sintomo, in questo caso il cancro duro, la paziente cerchi cure ospedaliere, quando riceve la diagnosi di sifilide primaria (AVELLEIRA et al, 2006).

II maggior numero di morti nelle donne incinte con sifilide era dovuto a un'altra causa. Secondo la letteratura più della metà delle morti materne in tutto il mondo erano per emorragia, ipertensione e setticemia (SAY, 2014).

\section{CONCLUSIONE}

II maggior numero di test consente un trattamento migliore e una riduzione del numero di casi. Tuttavia, l'assenza di cure preventive durante le relazioni sessuali induce un aumento dei casi, specialmente tra le donne in età riproduttiva.

RC: 68388

Disponibile in: https://www.nucleodoconhecimento.com.br/salute/donne-incinte 
Le aree con una popolazione più numerosa hanno un numero maggiore di casi. L'elevata variazione di partner in queste aree determina una maggiore contaminazione.

Il tipo di test influenza i numeri. Test meno specifici e più generali trovano anticorpi specifici e non specifici per il batterio. Questo può cambiare i numeri effettivi. L'assistenza sanitaria femminile rende precoce la diagnosi, facilitando il trattamento.

La sifilide non è direttamente responsabile del maggior numero di mortalità nelle donne in gravidanza.

\section{RIFERIMENTI}

AVELLEITA, J. C. R.; BOTTINO, G; Sífilis: Diagnóstico, Tratamento e Controle. An. Bras. Dermatol, Vol.81 no.2 Rio de Janeiro Mar./Apr. 2006.

BRASIL São Paulo, Centro de Referência e Treinamento DST/Aids. Boletim Epidemiológico. Ano XXX. 2013.

BRASIL, Ministério da Saúde. Secretaria de Ciência, Tecnologia e Insumos Estratégicos. Relatório de Recomendação, n¹59, 2015. Disponível em: <file:///C:/Users/aluno.SELABI/Desktop/CURSO\%20DE\%20ESCRITA/(BRASIL,\%20 2015).pdf>. Acesso em:26/09/2017.

BRASIL. Ministério da Saúde Secretaria de Vigilância em Saúde - Departamento de DST, Aids e Hepatites Virais SAF SUL Trecho 2 Boletim Epidemiológico - Sífilis Ano IV- no 1, 2015.

BRASIL. Ministério da Saúde. Ministério da Saúde Lança Campanha de Combate à Sífilis. Disponível em: <http://www.brasil.gov.br/saude/2013/10/ministerio-dasaude-lanca-campanha-de-combate-a-sifilis>. Acesso em: 19/09/2017.

$\mathrm{RC}: 68388$

Disponibile in: https://www.nucleodoconhecimento.com.br/salute/donne-incinte 
BRASIL. Ministério da Saúde. Portal da Saúde. Combate á Sífilis Congênita. Disponivel em: <http://portalarquivos.saude.gov.br/campanhas/sifilis/>. Acesso em: 19/09/2017.

BRASIL. Ministério da Saúde. Secretaria de Vigilância em Saúde. Departamento de DST, Aids e Hepatites Virais. Pesquisa de conhecimento, atitudes e práticas na população brasileira / Ministério da Saúde. Secretaria de Vigilância em Saúde. Departamento de DST, Aids e Hepatites Virais. - Brasília: Ministério da Saúde, 2011.

BRASIL. Ministério da Saúde. Secretaria de Vigilância em Saúde. Departamento de DST, Aids e Hepatites Virais. Pesquisa de conhecimento, atitudes e práticas na população brasileira / Ministério da Saúde. Secretaria de Vigilância em Saúde. Departamento de DST, Aids e Hepatites Virais. - Brasília: Ministério da Saúde, 2011.

BRETAS, J. R. S.; OHARA, C. V. S.; JARDIM, D. P.; MUROYA, R. L.; Conhecimento Sobre DST/AIDS por Estudantes Adolescentes. Rev. esc. enferm. USP vol.43 no.3 São Paulo Sept. 2009.

CARVALHO, I. S.; BRITO, R. S; Sífilis congênita no Rio Grande do Norte: estudo descritivo do período 2007-2010. Epidemiol. Serv. Saúde, v.23 n.2 Brasília, 2014.

COSTA, C. C.; FEITAS, L. V.; SOUSA, D. M. N.; OLIVEIRA, L. L.; CHAGAS, A. C. M. A.; LOPES, M. V. O.; DAMASCENO, A. K. C. Sífilis Congênita No Ceará: Análise Epidemiológica De Uma Década. Rev Esc Enferm USP 2013; 47(1):149-56.

IBGE, Censo Demográfico 1960, 1970, 1980, 1991, 2000 e 2010. Disponível em: $<$ https://censo2010.ibge.gov.br/sinopse/index.php?dados=8>. Acesso em: 26/09/2017.

JUNIOR, W. B.; SHIRATSU, R.; PINTO, V.; Abordagem nas Doenças Sexualmente Transmissíveis. An Bras Dermatol. 2009;84(2):151-59.

RC: 68388

Disponibile in: https://www.nucleodoconhecimento.com.br/salute/donne-incinte 
KALININ, Y.; NETO, A. P.; PASSARELLI, D. H. C. Sífilis: aspectos clínicos, transmissão, Manifestações orais, diagnóstico e tratamento. Odonto 2015; 23(4546): 65-76.

MESQUITA, K.; LIMA, G. K.; FILGUEIRA, A.; FLÔR, S. M.; FREITAS, C. A.; LINHARES, M. S.; GUBERT, F.Análise dos Casos de Sífilis Congênita em Sobral, Ceará: Contribuições para Assistência Pré-Natal. DST - J bras Doenças Sex Transm 2012; 24(1):20-27

ROCHA, K.D., FECURY, A.A., OLIVEIRA, E., DENDASCK, C.V., DIAS, C.A.G.M. Number of congenital syphilis cases in Brazil between 2009 and 2013. Revista Científica Multidisciplinar Núcleo do Conhecimento. Year 05, Ed. 05, Vol. 01, pp. 131-143. May 2020. ISSN:2448-0959.

DOI:

10.32749/nucleodoconhecimento.com.br/health/congenital-syphilis-cases.

SAY, L.; CHAU, D.; GEMMILL, A.; TUBÇALP, O.; MOLLER, A.; DANIELS, J.; GULMEZOGLU, A. M.; TEMMERMAN, M.; ALKEMA, L. Global Causes Of Maternal Death: A Who Systematic Analysis. Lancet Glob Health, Vol.2, 2014.

SUTO, C. S. S.; SILVA, D. L.; ALMEIDA, E. S.; COSTA, E. L.; EVANGELISTA, T. J. Assistência Pré-Natal A Gestante Com Diagnóstico De Sífilis Revista de Enfermagem e Atenção à Saúde 2016; 5(2): 18-33.

Inviato: Novembre 2020.

Approvato: Novembre 2020.

RC: 68388

Disponibile in: https://www.nucleodoconhecimento.com.br/salute/donne-incinte 